\title{
Tumor-penetrating peptide enhances transcytosis of silicasome-based chemotherapy for pancreatic cancer
}

\author{
Xiangsheng Liu, ${ }^{1}$ Paulina Lin, ${ }^{1}$ lan Perrett, ${ }^{1}$ Joshua Lin, ${ }^{1}$ Yu-Pei Liao, ${ }^{1}$ Chong Hyun Chang, ${ }^{1}$ Jinhong Jiang, ${ }^{1}$ Nanping Wu, ${ }^{2}$ \\ Timothy Donahue, ${ }^{2}$ Zev Wainberg, ${ }^{3}$ Andre E. Nel, ${ }^{1,4}$ and Huan Meng ${ }^{1,4}$ \\ 'Department of Medicine, Division of NanoMedicine, 'Department of Surgery, Division of Ceneral Surgery, and Department of Molecular and Medical Pharmacology, David Geffen School of Medicine at UCLA, \\ ${ }^{3}$ Department of Medicine, and ${ }^{4}$ California NanoSystems Institute, University of California, Los Angeles, California, USA.
}

\begin{abstract}
Pancreatic ductal adenocarcinoma (PDAC) is almost uniformly fatal; however, some improvement in overall survival has been achieved with the introduction of nanocarriers that deliver irinotecan or paclitaxel. Although it is generally assumed that nanocarriers rely principally on abnormal leaky vasculature for tumor access, a transcytosis transport pathway that is regulated by neuropilin-1 (NRP-1) has recently been reported. NRP-1-mediated transport can be triggered by the cyclic tumor-penetrating peptide iRCD. In a KRAS-induced orthotopic PDAC model, coadministration of iRCD enhanced the uptake of an irinotecan-loaded silicasome carrier that comprises lipid bilayer-coated mesoporous silica nanoparticles (MSNPs); this uptake resulted in enhanced survival and markedly reduced metastasis. Further, ultrastructural imaging of the treated tumors revealed that iRCD coadministration induced a vesicular transport pathway that carried Au-labeled silicacomes from the blood vessel lumen to a perinuclear site within cancer cells. iRCD-mediated enhancement of silicasome uptake was also observed in patient-derived xenografts, commensurate with the level of NRP-1 expression on tumor blood vessels. These results demonstrate that iRCD enhances the efficacy of irinotecan-loaded silicasome-based therapy and may be a suitable adjuvant in nanoparticle-based treatments for PDAC.
\end{abstract}

\section{Introduction}

The emergence of nanotechnology has improved cancer chemotherapy over the last decade (1-6). This also holds true for pancreatic ductal adenocarcinoma (PDAC), which in spite of its poor prognosis, has shown modest improvement ( 2 months) in overall survival (OS) with the introduction of 2 FDA-approved carriers, Abraxane and Onivyde. The Abraxane effect reflects the ability of this albumin carrier to deliver paclitaxel, which enhances gemcitabine uptake, while the liposomal carrier (Onivyde) improves the pharmacokinetics of irinotecan delivery $(7,8)$. However, in spite of the modest improvement in OS (7-11), a key challenge for delivery of chemotherapy by nanocarriers remains restricted vascular access due to vascular abnormalities and a desmoplastic stroma that contributes to dense pericyte coverage of vascular fenestrations $(12,13)$. In fact, the blocked egress of nanoparticles from the PDAC vascular bed does not conform to the idea of an enhanced permeability and retention (EPR) effect resulting from leaky blood vessels (12-15). We have previously demonstrated that stromal-vascular engineering by a nanocarrier, which delivers a small molecule inhibitor of the TGF- $\beta$ receptor signaling pathway, is capable of improving vascular egress of gemcitabine-loaded liposomes in PDAC as a result of interfering with pericyte adherence to endothelial cells (16).

\section{Related Commentary: p. 1622}

Conflict of interest: The authors have declared that no conflict of interest exists. Submitted: December 19, 2016; Accepted: February 2, 2017 Reference information: J Clin Invest. 2017;127(5):2007-2018. https://doi.org/10.1172/JCI92284.
Another important recent advance has been the discovery of a vascular transcytosis pathway that allows nanocarrier uptake at the tumor site by a mechanism that differs from the EPR effect (1721). It has been demonstrated that this pathway can be initiated by the cyclic tumor-penetrating peptide iRGD (CRGDK/RGPD/ EC), which interacts with the neuropilin-1 (NRP-1) receptor (17, 19, 20). NRP-1 plays a key role in tumor angiogenesis and can also be engaged by VEGFs that contain an RXXR sequence, also dubbed the C-terminal rule (CendR) motif $(18,21)$. The transcytosis pathway is not only distinct from other endocytic uptake mechanisms (e.g., caveolae, clathrin-coated pits, and macropinocytosis), but it also allows entry of small drug molecules, monoclonal antibodies (e.g., trastuzumab), and nanoparticles (e.g., Abraxane and doxorubicin liposomes) during iRGD coadministration $(19,21)$. Importantly, this effect is not dependent on direct conjugation of the peptide to the drug or carrier, as demonstrated by gemcitabine uptake in some (but not all) experimental PDAC models (22). However, no detailed investigation of the PDAC transcytosis pathway has been undertaken, and it is unknown whether it could be beneficial from the perspective of iRGD peptide coadministration to further enhance the nanocarrier OS effects $(7,8)$.

We have developed a multifunctional mesoporous silica nanoparticle (MSNP) platform $(16,23-25)$ that has recently been adapted to provide high-dose PDAC chemotherapy using a supported lipid bilayer (LB) for drug encapsulation $(26,27)$. This carrier has also been designated as a "silicasome" to distinguish it from morphologically similar liposomal carriers, which contain a nonsupported LB. However, compared with liposomes, including an in-house liposomal carrier for the irinotecan, silicasomes have 

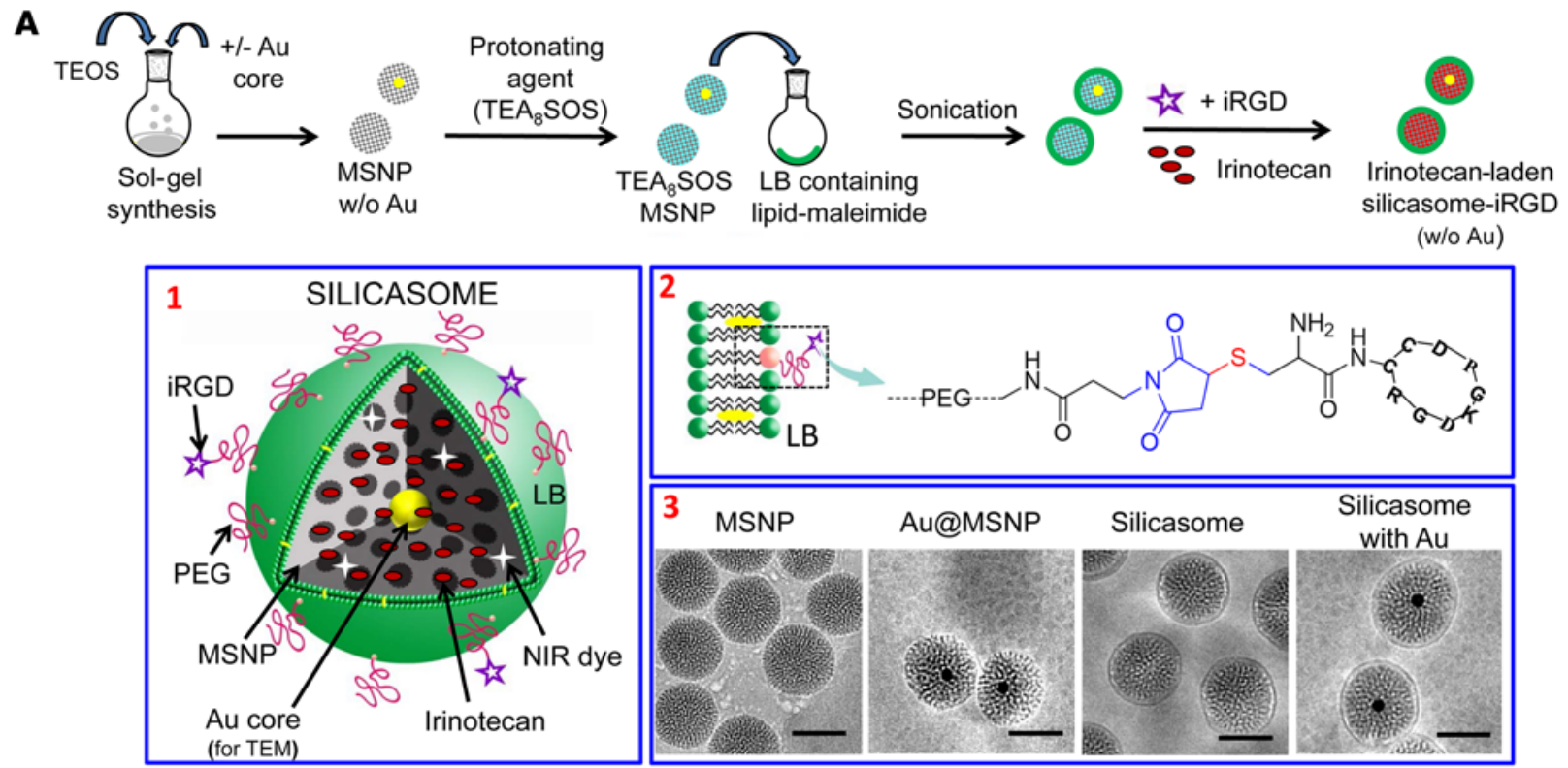

B
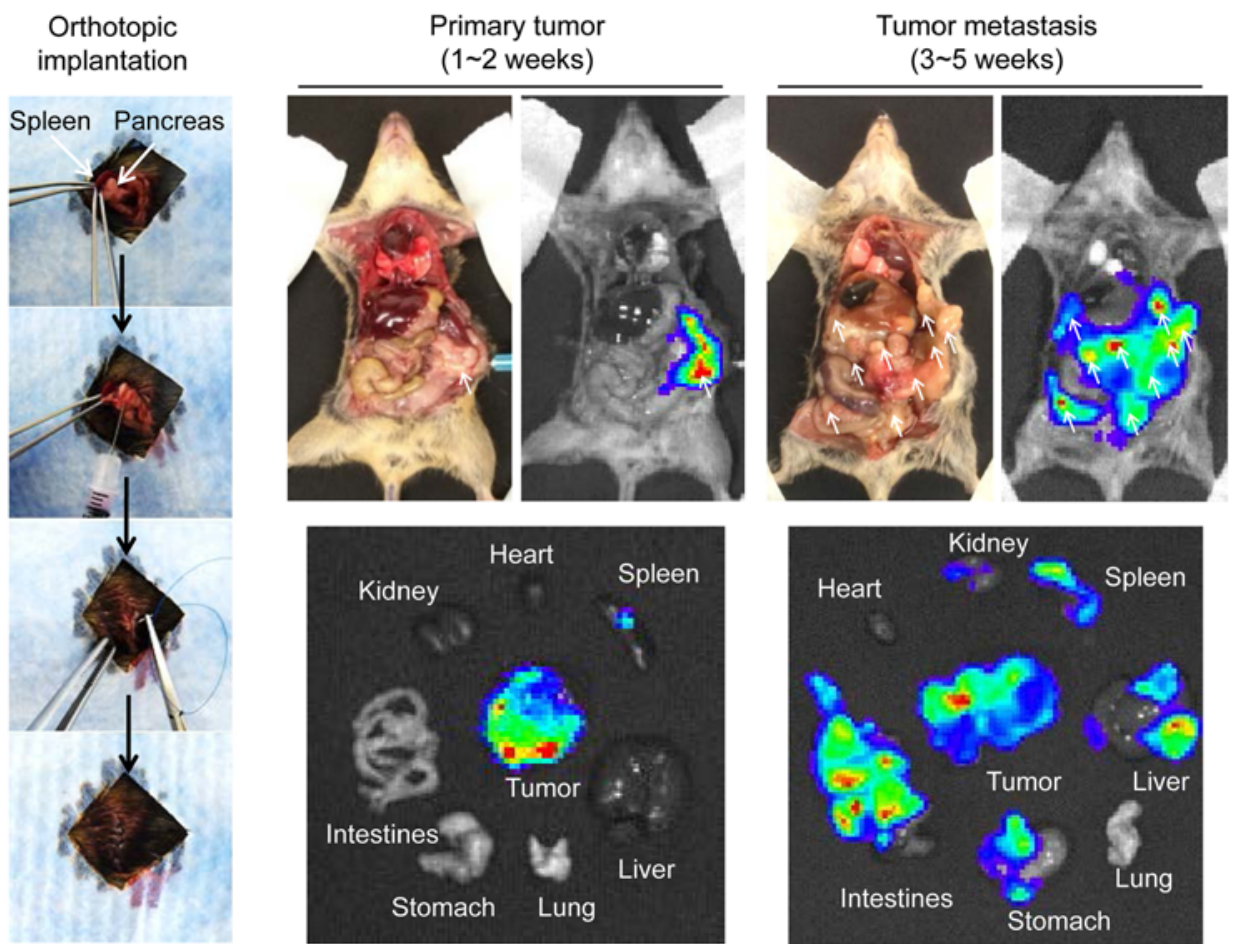

$\sim 30$ min surgery

Figure 1. Synthesis and characterization of silicasomes for drug loading and visualization. (A) The top panel provides a schematic that shows the synthesis steps for constructing silicasomes and remote drug loading $(26,27)$. Briefly, MSNP cores were synthesized by sol-gel chemistry and soaked in a solution containing the protonating agent, $\mathrm{TEA}_{8} \mathrm{SOS}$. These particles were coated with an LB, using a sonication procedure in the presence of a lipid biofilm (27). This was followed by remote irinotecan loading across the proton gradient provided by TEA ${ }_{8}$ SOS. Box 1: schematic of the different silicasome components. Box 2: iRGD peptide conjugation to the LB, using a thiol-maleimide reaction to link the cysteine-modified iRGD peptide to DSPE-PEG ${ }_{2000}-\mathrm{maleim}$ ide. Box 3: cryoEM images to show the bare particles and the silicasomes, with and without the embedding of approximately $10 \mathrm{~nm}$ Au cores (for TEM visualization). Synthesis procedures are described in Supplemental Materials and Methods. Scale bars: $50 \mathrm{~nm}$. (B) Autopsy and IVIS images of the KPC-derived orthotopic PDAC model in immunocompetent B6/129 mice. The orthotopic implantation involves minor surgery to inject $2 \times 10^{6} \mathrm{KPC}-$ luc cells in the tail of the pancreas (left panel). The autopsy and bioluminescence imaging reveal primary tumor growth after 1 to 2 weeks, followed by tumor metastases by 3 to 5 weeks. Macrometastases are marked by arrows. 
Table 1. A summary of physicochemical properties of the silicasomes used in this study

\begin{tabular}{ll}
\multicolumn{3}{l}{ Silicasome physicochemical properties } \\
MSNP surface area (BET) & $\sim 850 \mathrm{~m}^{2} / \mathrm{g}$ \\
MSNP pore volume (BET) & $\sim 0.75 \mathrm{~cm}^{3} / \mathrm{g}$ \\
MSNP pore size (BET, TEM) & $\sim 3 \mathrm{~nm}$ \\
MSNP core size (TEM) & $\sim 65 \mathrm{~nm}$ \\
LB thickness (cryoEM) & $\sim 7 \mathrm{~nm}$ \\
Silicasome size (cryoEM) & $\sim 80 \mathrm{~nm}$ \\
Hydrodynamic size (DLS) & $\sim 130 \mathrm{~nm}$ \\
(polydispersity index) & (PDI 0.08) \\
$\zeta$ Potential & $\sim-10 \mathrm{mV}$ \\
Drug-loading capacity & $\sim 50 \%$ \\
(irinotecan) & (w/w, drug/MSNP)
\end{tabular}

BET, Brunauer-Emmett-Teller; DLS, dynamic light scattering.

been shown to exhibit a significantly higher drug-loading capacity, improved circulatory stability (as a result of the supported LB), and reduced drug leakage (27). These features allow for improved pharmacokinetics and treatment efficacy of silicasomes versus liposomes in a stringent orthotopic Kras PDAC model developed by using KPC cells (derived from a spontaneous PDAC tumor from a transgenic $\mathrm{Kras}^{\mathrm{LSL}-\mathrm{G} 12 \mathrm{D} /+} \mathrm{Tr} p 53^{\mathrm{LSL}-\mathrm{R} 172 \mathrm{H} /+} \mathrm{Pdx1}$-Cre mouse) (27-29). In addition, silicasomes also provide major toxicity reduction in the gastrointestinal tract, liver, and bone marrow compared with the liposomal equivalent (27). Besides the success with irinotecan, the silicasome platform has also been adapted for synergistic delivery of paclitaxel and gemcitabine, allowing it to significantly (>10-fold) outperform the combination of free gemcitabine with Abraxane in an orthotopic PDAC model (26). It is noteworthy that the above results with silicasome carriers could be achieved by "passive" delivery without the need to resort to targeting ligands.

Given this background, we were interested in the impact of transcytosis on the efficacy of PDAC treatment by irinotecan-loaded silicasomes and whether transcytosis is best accomplished by iRGD conjugation to the carrier or its coadministration. We demonstrate the feasibility of using the coadministration of free iRGD peptide to enhance carrier uptake and treatment efficacy in a Kras orthotopic model. Moreover, we provide ultrastructural evidence of a grouped vesicle system that allows transport of $\mathrm{Au}$-labeled silicasomes from the blood vessel lumen to a perinuclear site in cancer cells. We also demonstrate in patient-derived PDAC xenografts that the relative abundance of NRP-1 expression on the tumor vasculature determines the response magnitude to silicasomes through the use of iRGD coadministration. All considered, these data demonstrate an important nanocarrier delivery pathway in addition to the traditional reliance on increased vascular permeability at the tumor site.

\section{Results}

Synthesis and characterization of silicasomes for drug loading and visualization in PDAC tumors. We previously demonstrated high irinotecan loading by an LB-coated MSNP (also known as a silicasome) using a remote loading technique that relies on a protonating agent (Figure 1A) (27). Irinotecan is a weak basic and amphi- pathic molecule that can diffuse across the LB into the porous MSNP packaging space, where proton release by prior entrapped triethylammonium sucrose octasulfate (TEA $\mathrm{TOS}_{8}$ ) converts the drug into a hydrophilic derivative, incapable of back diffusion across the LB (Figure 1A). The remote loading procedure was used to synthesize an irinotecan-loaded silicasome batch that achieved a drug-loading capacity of approximately $50 \%$ by weight (50 wt $\%$ ) (\% weight per weight $[\mathrm{w} / \mathrm{w}]$, irinotecan/MSNP) (27). To determine whether conjugation of an iRGD peptide onto the silicasome surface can affect carrier biodistribution to the PDAC tumor site, we also synthesized a particle batch in which the DSPE-PEG ${ }_{2000}$ component of the LB was used for conjugation to a cysteine residue in the peptide. This was accomplished by substituting DSPEPEG $_{2000}$ with DSPE-PEG 2000 -maleimide (see Methods), allowing thiol-maleimide coupling to the cysteine-modified peptide Cys-c (CRGDKGPDC) (Figure 1A). To confirm the success of the conjugation reaction, we used a FAM-labeled version of the peptide (FAM-iRGD) developed by Sugahara et al. to perform fluorescence spectroscopy of conjugated silicasomes after extensive washing (Supplemental Figure 1A; supplemental material available online with this article; https://oi.org/10.1172/JCI92284DS1) (17). This confirmed the stable association of the fluorescent peptide with the LB. The density of iRGD conjugation was limited to approximately 3 molar percentage ( $\mathrm{mol} \%$ ) (of all LB components) to prevent colloidal instability and interference in carrier uptake. The uptake of the intact silicasome-iRGD carrier was confirmed by flow cytometry and confocal microscopy in KPC cells (Supplemental Figure 1, B and C).

In order to perform transmission electron microscopy (TEM) visualization of the transcytosis process in PDAC tumors, we also synthesized a batch of core-shell MSNPs that included approximately $10 \mathrm{~nm}$ electron-dense Au-nanoparticles (Figure 1A). Similarly to the bare particles, the core/shell particles could be effectively coated with an LB, as demonstrated by cryo-electron microscopy (CryoEM) (Figure 1A). The detailed synthesis and characterization procedures for all the carriers used in this communication are discussed in Supplemental Materials and Methods. The main physicochemical characteristics of silicasomes are summarized in Table 1.

Comparison of the effect of conjugated versus nonconjugated $i R G D$ peptide on silicasome biodistribution in an orthotopic PDAC model. Luciferase-expressing KPC cells derived from a spontaneous PDAC tumor from a transgenic $\mathrm{Kras}^{\mathrm{LSL}-\mathrm{G12D} /{ }^{+}} \operatorname{Trp} 53^{\mathrm{LSL}-\mathrm{R} 172 \mathrm{H} /{ }^{+}} \mathrm{Pd} \mathrm{d} 1-$ Cre animal were orthotopically implanted in the pancreas tails in immunocompetent B6/129 mice (Figure 1B) $(27,28)$. This stringent PDAC tumor model mimics human PDAC for oncogene expression, growth characteristics, metastasis, histological features, and development of a dysplastic stroma $(28,29)$. Figure 1 B summarizes the details of the tumor growth characteristics and metastasis as seen during animal autopsy and IVIS imaging. Silicasome biodistribution to the orthotopic tumor site was assessed by a one-time i.v. injection of $50 \mathrm{mg} / \mathrm{kg}$ near-infrared (NIR) dye-labeled (DyLight 680) particles that were either nonconjugated (i.e., iRGD-free status) or peptide conjugated (silicasome-iRGD) (Figure 2A). A third group of animals received coadministration of $8 \mu \mathrm{mol} / \mathrm{kg}$ free peptide plus nonconjugated particles (silicasome + iRGD). IVIS imaging of the explanted organs, performed 24 hours after initial 
A

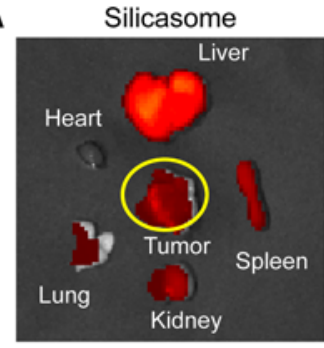

Silicasome-iRGD

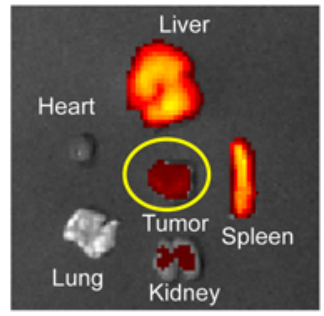

Silicasome + iRGD

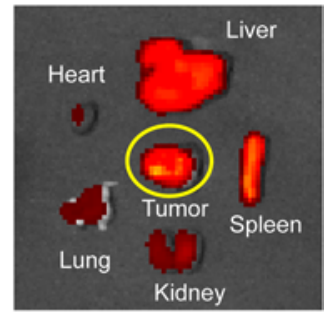

B

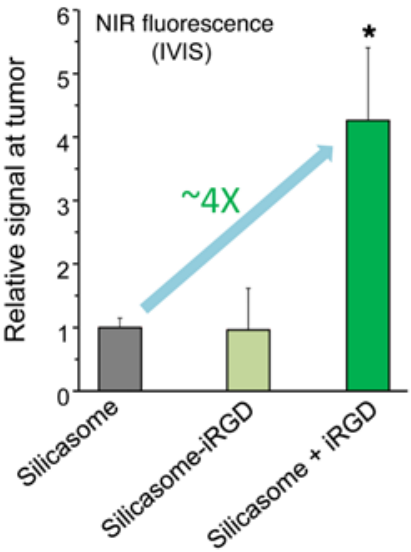

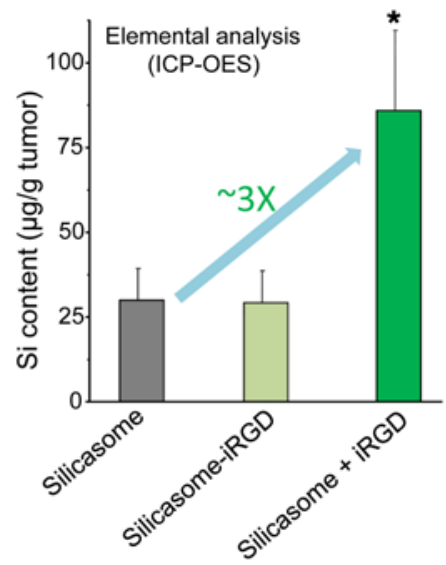

Figure 2. Coadministrated iRGD enhanced the tumor biodistribution of i.v.-injected silicasomes in the KPC-derived orthotopic model. (A) The tumor-bearing mice received (a) 50 $\mathrm{mg} / \mathrm{kg}$ of NIR-labeled silicasome i.v. with coadministration of $8 \mu \mathrm{mol} / \mathrm{kg}$ free iRGD ( $n=3$, referred to as silicasome + iRGD), (b) $50 \mathrm{mg} / \mathrm{kg}$ of the NIR-labeled, iRGD-conjugated silicasome ( $n=3$, referred to as silicasome-iRGD), or (c) $50 \mathrm{mg} / \mathrm{kg} \mathrm{NIR-la-}$ beled silicasome without $\operatorname{RRCD}(n=3)$. Animals were sacrificed 24 hours after injection, followed by ex vivo NIR imaging using IVIS. (B) NIR fluorescence intensity and Si content were used to quantify the nanoparticle content in the orthotopic tumors. Data represent mean $\pm S D$. ${ }^{*} P<0.05$, 1-way ANOVA followed by Tukey's test. This experiment was repeated twice. injection and animal sacrifice, demonstrated a prominent increase in the NIR-signaling intensity at the tumor site for the silicasome + iRGD group compared with the signaling intensity in the silicasome-iRGD or silicasome-only groups (Figure 2A). Coadministration of free iRGD did not significantly influence the particle distribution in normal organs. Imaging intensity was quantified by IVIS Lumina Living Image software. In contrast to their lack of an in vivo effect, the peptide-conjugated silicasomes could be seen to enhance carrier uptake in KPC cells (Supplemental Figure 1, B and $\mathrm{C}$ ). We interpret that as sufficient NRP-1 receptor density to initiate transmembrane uptake, while the receptor abundance at the tumor vascular site may be more limiting to the number of conjugated particles that dock and are allowed through, as previously reported for in vitro/in vivo comparisons by Ruoslahti et al. (17, 18,30 ). In order to show that the NIR intensity (Figure $2 \mathrm{~B}$ ) reflects actual MSNP uptake, inductively coupled plasma optical emission spectrometry (ICP-OES) was used for quantification of the tumor Si content (Figure 2B). This demonstrated a significant ( 3 -fold) increase in Si content in the silicasome + iRGD group compared with the silicasome-iRGD or the silicasome-only groups. We also demonstrated the importance of the CendR motif for PDAC biodistribution by performing a separate experiment to show that silicasome coadministration with a control non-CendR peptide, cyclo (RGDfK) (19), was incapable of enhancing silicasome uptake (Supplemental Figure 2). This result was also confirmed by ICP-OES (Supplemental Figure 2).

Tumor tissue sections were used to assess the relative abundance of intratumor biodistribution of NIR-labeled silicasomes and traveling distance from the tumor blood vessels, which were stained with Alexa Fluor 488-conjugated anti-CD31 (Supplemental Figure 3). This analysis showed that iRGD coadministration was the most effective strategy for enhancing silicasome uptake at orthotopic tumor sites. In contrast, the iRGD-conjugated carrier failed to exert a significant effect on the number of particles as well as the traveling distance in our KPC orthotopic model (Supplemental Figure 3). The above findings pave the way for choosing free rather than conjugated iRGD to perform subsequent efficacy studies.

Because all nanocarriers are also taken up in the reticuloendothelial system (RES), it is important to comment on the silicasome uptake in the liver and spleen (with little appreciable effects on the lungs, hearts, and kidneys), as seen in Figure 2A. Interestingly, IVIS imaging showed a possible increase in the biodistribution of the conjugated carrier to the RES organs. This was confirmed by an increased Si content in the liver and spleen during performance of ICP-OES (Supplemental Figure 4). While we lack an exact explanation for this observation, it is possible that peptide conjugation, directly or indirectly, leads to particle opsonization and increased uptake by scavenger receptors, independently of NRP-1 (31).

$i R G D$ coadministration enhances the efficacy of irinotecan delivery by silicasome. To demonstrate the possible therapeutic benefit of iRGD coadministration in PDAC treatment with the irinotecan-loaded silicasome, an efficacy experiment was performed in the same orthotopic tumor model. Animals received i.v. injection of the irinotecan-loaded silicasomes at a drug dose of $40 \mathrm{mg} / \mathrm{kg}$ (equivalent to carrier dose of $80 \mathrm{mg} / \mathrm{kg}$ ) with or without coadministration of $8 \mu \mathrm{mol} / \mathrm{kg}$ iRGD. Treatment commenced 13 days after orthotopic implantation of $2 \times 10^{6} \mathrm{KPC}$-luc cells (Figure $3 \mathrm{~A}$ ), at which time the primary tumor size was approximately 3 to $5 \mathrm{~mm}$ in the absence of macrometastasis (27). Injections were repeated every 3 days, for a total of 4 administrations (Figure 3A). The control groups consisted of animals receiving i.v. PBS, the irinotecan-loaded silicasome alone (same dose), or free iRGD alone (same dose). Kaplan-Meier plots were used to express animal survival $(27,32)$, and animal autopsy was used to assess local tumor 
A

Tumor
implantation
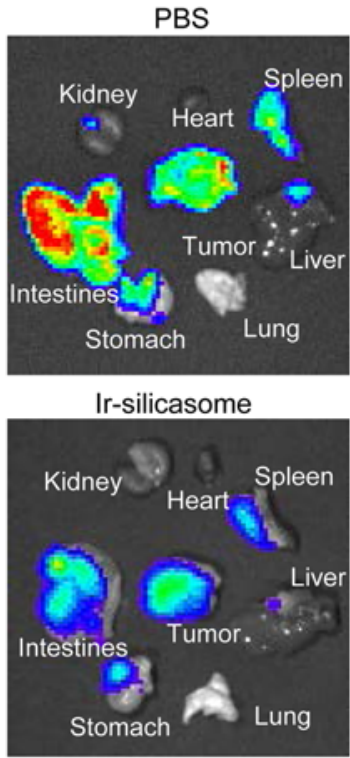

Free iRGD

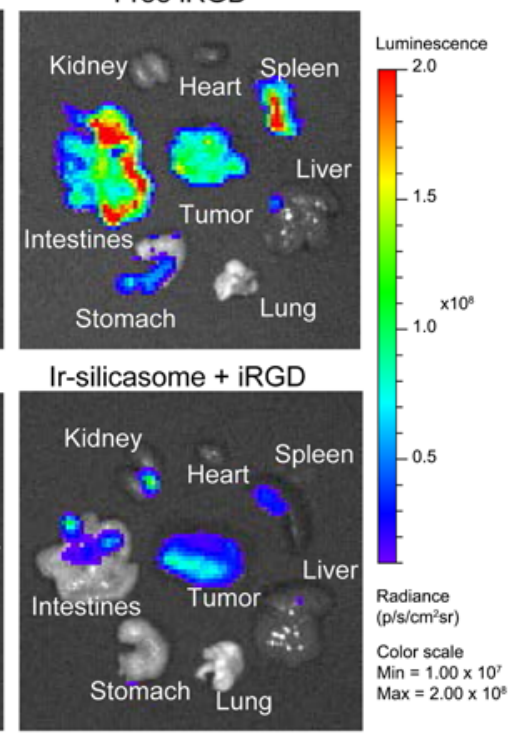

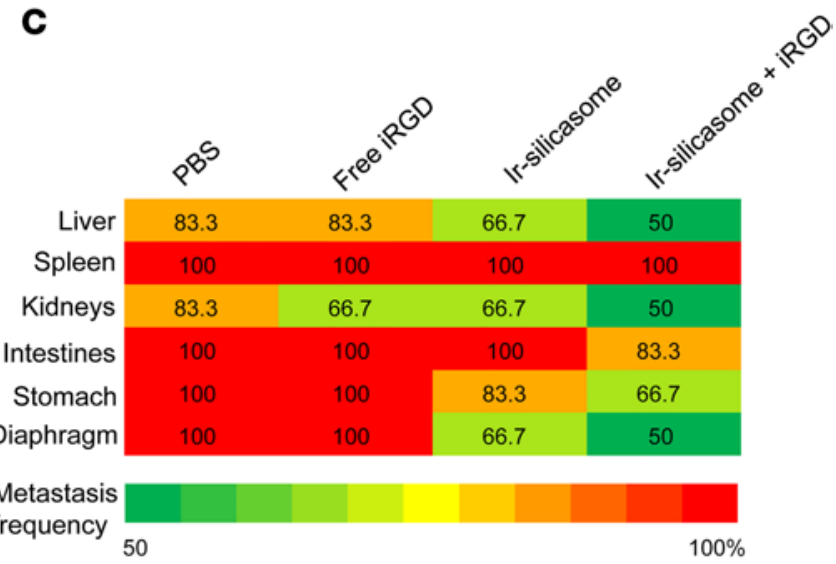

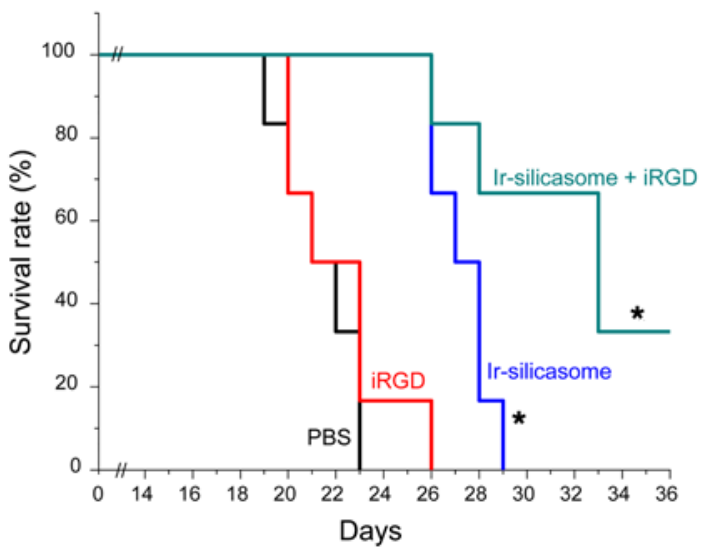

E

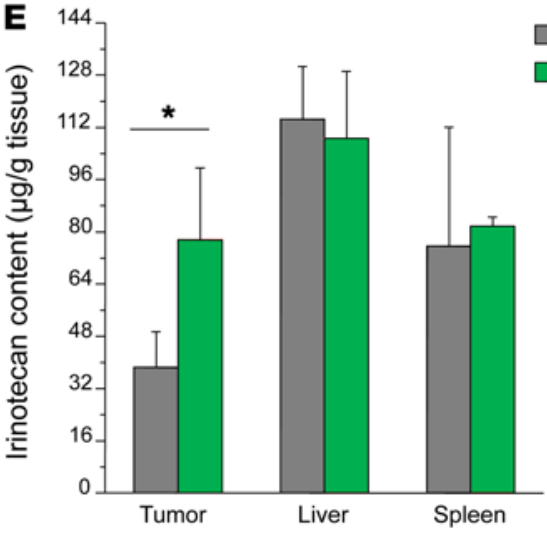

-silicasome

Ir-silicasome + iRGD

Figure 3. iRGD coadministration enhances the uptake and efficacy of irinotecan-loaded silicasome in the KPC-derived orthotopic model. (A) Schedule of the efficacy study in the luciferase-expressing KPC-derived orthotopic tumors $(n=6)$. The chosen irinotecan-loaded silicasome dose $(40 \mathrm{mg} / \mathrm{kg}$ irinotecan; $80 \mathrm{mg} / \mathrm{kg}$ MSNP) is based on a previous efficacy study (27). This dose of the irinotecan-loaded silicasome was i.v. injected, with or without coadministration of $8 \mu \mathrm{mol} / \mathrm{kg}$ iRGD. The injections were repeated every 3 days, for a total of 4 administrations. The controls involved animal groups receiving identical doses of free iRGD or the irinotecan-loaded silicasome alone. (B) Representative ex vivo imaging of the bioluminescence intensity in the mice prior to sacrifice to show the primary tumor burden and metastases. The images show that iRGD coadministration could enhance the silicasome efficacy. Ir-silicasome, irinotecan-loaded silicasome. (C) Heat map summarizing the impact on tumor and tumor metastasis inhibition of the experiment shown in B. (D) iRGD coadministration improved the survival impact of the irinotecan-loaded silicasome, as shown by Kaplan-Meier analysis. The effect of silicasome alone is highly significant compared with that of PBS and free $\operatorname{iRGD~}(P=0.001$, log-rank test). iRGD coadministration further enhances survival $(P=0.027$, log-rank test). (E) HPLC analysis of the irinotecan content in the tumor 24 hours after a one-time dose of irinotecan-loaded silicasome (40 mg/kg drug) was injected, with or without coadministration of $8 \mu \mathrm{mol} / \mathrm{kg}$ iRGD. Data represent mean \pm SD $(n=3) .{ }^{*} P<0.05,2$-tailed Student's $t$ test.

spread and the presence of metastasis. Numerous metastatic foci could be seen in the spleens, intestines, stomachs, livers, and kidneys of animals treated with saline or free iRGD peptide (Figure 3B). While the irinotecan-loaded silicasome significantly reduced the tumor burden and number of metastases, iRGD coadministration further enhanced shrinking of the primary tumor as well as inhibiting the spread to the liver, stomach, and intestines (Figure $3 \mathrm{~B})$. The heat map in Figure $3 \mathrm{C}$ provides a quantitative display of the impact of the coadministered peptide on metastatic disease. In addition, log-rank testing (SPSS 19.0 software, IBM SPSS Statistics) also demonstrated that, compared with the PBS group, treatment with the irinotecan-loaded silicasome alone improved 
A

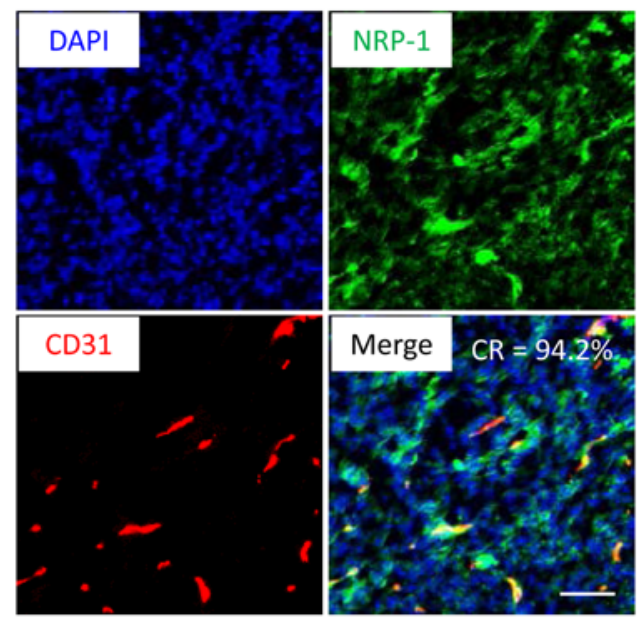

B

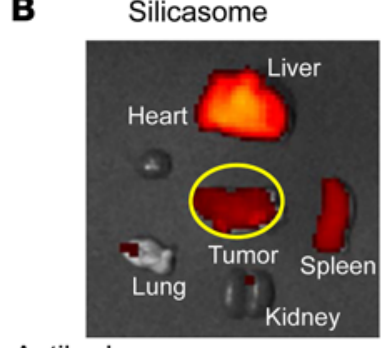

Antibody:
$-$
Silicasome + iRGD

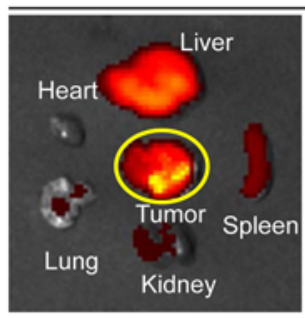

Control IgG

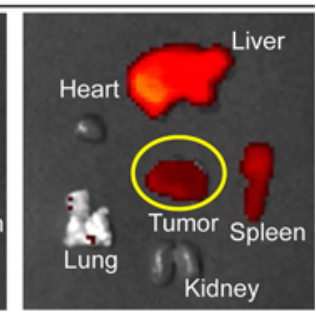

Anti-NRP-1
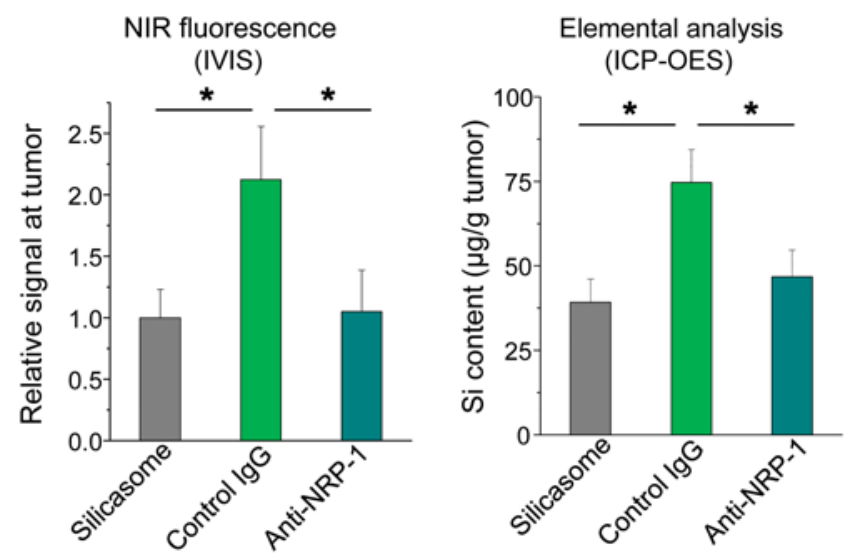

Figure 4. IRGD-mediated silicasome uptake requires NRP-1 expression on the tumor vasculature. (A) Multicolor IHC staining of NRP-1 (green) and CD31 (red), plus nuclear staining (blue) in a KPC-derived tumor section. The IHC staining methodology is described in Methods. NRP-1 is expressed on the tumor tissue as well the blood vessels. The merged image shows a high degree of colocalization (94.2\%) of NRP-1 with CD31; the colocalization ratio (CR) was determined by Imagel software. Scale bar: $100 \mu \mathrm{m}$. (B) Interference of an anti-NRP-1 antibody on iRGD-mediated silicasome biodistribution. Fifteen minutes before i.v. injection of $50 \mathrm{mg} / \mathrm{kg}$ of the NIR-silicasome $+8 \mu \mathrm{mol} / \mathrm{kg}$ free iRGD $(n=3), 50 \mu \mathrm{g}$ of the blocking antibody or a control lgG was i.v. injected. Ex vivo NIR images showing nanoparticle biodistribution after 24 hours. NIR intensity as well as Si content was used to quantify the nanoparticle uptake at the orthotopic tumor site. Data represent mean \pm SD. ${ }^{*} P<0.05$, 1-way ANOVA followed by Tukey's test.

survival by $28.6 \%$ compared with $57.1 \%$ during coadministration of the iRGD peptide (Figure 3D); this difference is statistically significant $(P=0.027)$. We also confirmed that there was a significant ( 2 -fold) increase in the intratumoral content of irinotecan in animals receiving a one-time administration of iRGD $(8 \mu \mathrm{mol} / \mathrm{kg})$ i.v. together with irinotecan-loaded silicasomes ( $40 \mathrm{mg} / \mathrm{kg}$ drug), as determined by HPLC at 24 hours (Figure 3E).

iRGD-mediated silicasome uptake requires NRP-1 expression on the tumor vasculature. The mechanism of action of iRGD in mediating drug uptake is dependent on homing to $\alpha_{v} \beta_{3}$ or $\alpha_{v} \beta_{5}$ integrins, which are preferentially expressed on cancer blood vessels $(33,34)$. Binding of the cyclic peptide to the integrins is followed by cleavage and release of the C-terminal end of cleaved iRGD, which mediates the interaction with NRP-1 (this is also known as C-end rule). NRP-1 binding leads to the triggering of a system of vesicles that can assist drug and nanoparticle transport $(17,19,21)$. A schematic demonstrating the mechanism by which iRGD initiates nanoparticle transcytosis is shown in Supplemental Figure 5 (19). In order to determine the expression of NRP- 1 at the KPC tumor site, IHC staining was performed by Alexa Fluor 488-labeled anti-NRP-1, while endothelial cells and nuclei were localized by staining with Alexa Fluor 594-labeled anti-CD31 and DAPI, respectively. Fluorescent microscopy and Image (NIH) analysis were performed to determine percentage of overlap of NRP-1 with CD31; this demonstrated $94.2 \%$ colocalization (Figure $4 \mathrm{~A}$ ). To verify the role of NRP-1 in silicasome uptake at the orthotopic tumor site, tumor-bearing mice were preinjected with an antagonist antibody to the b1b2 domain of NRP-1 $(17,19)$. Subsequent administration of iRGD plus NIR-labeled silicasomes demonstrated a definitive reduction in the carrier uptake compared with that of animals not treated with a blocking antibody (Figure 4B). The same interference was not seen with the control IgG (Figure 4B). These data confirm the role of NRP-1 in iRGD-mediated silicasome uptake.

Ultrastructural demonstration of the transport of silicasomes by the transcytosis system at the KPC cancer site. It has been demonstrated that binding of the C-terminal end of iRGD to NRP-1 can initiate a bulk transcytosis pathway that involves a novel vesicular transport mechanism $(17,19,21)$. To the best of our knowledge, this transcytosis pathway has never been directly visualized during the transport of a therapeutic nanocarrier to the site of a tumor. Electron microscopy (EM), which could provide visual enhancement, was used for ultrastructural analysis of the iRGD-mediated transport pathway in the KPC model. Initially, we compared TEM images taken of the harvested tumor at different intervals after the i.v. injection of silicasomes, with or without iRGD coadministration (Figure 5A). At 24 hours, iRGD coadministration could clearly be seen to have induced the formation of grape-like vesicles, approximately 110 to $370 \mathrm{~nm}$ in diameter, that were spread across the endothelial cells, from the luminal to the abluminal aspect of the blood vessel (Figure 5A). These features resembled 
A

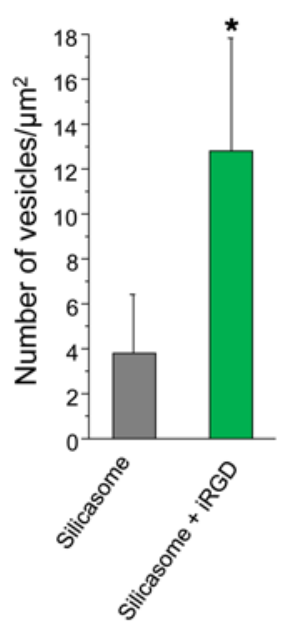

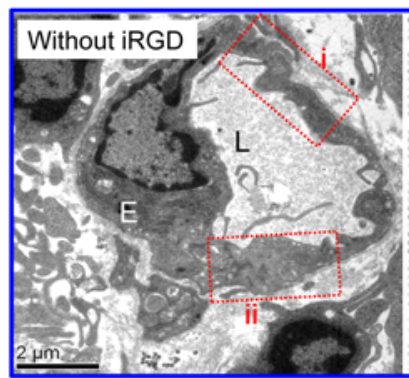
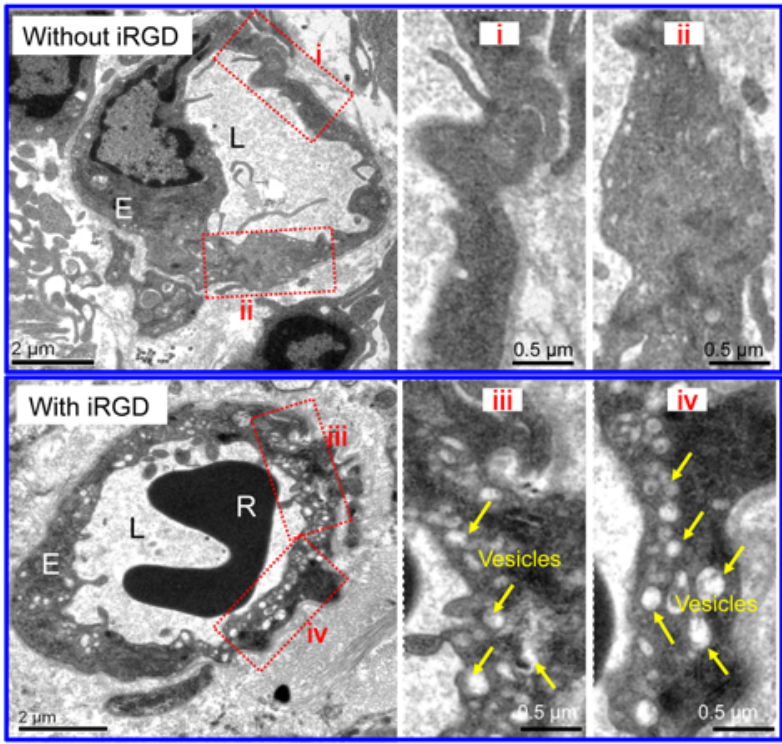

B
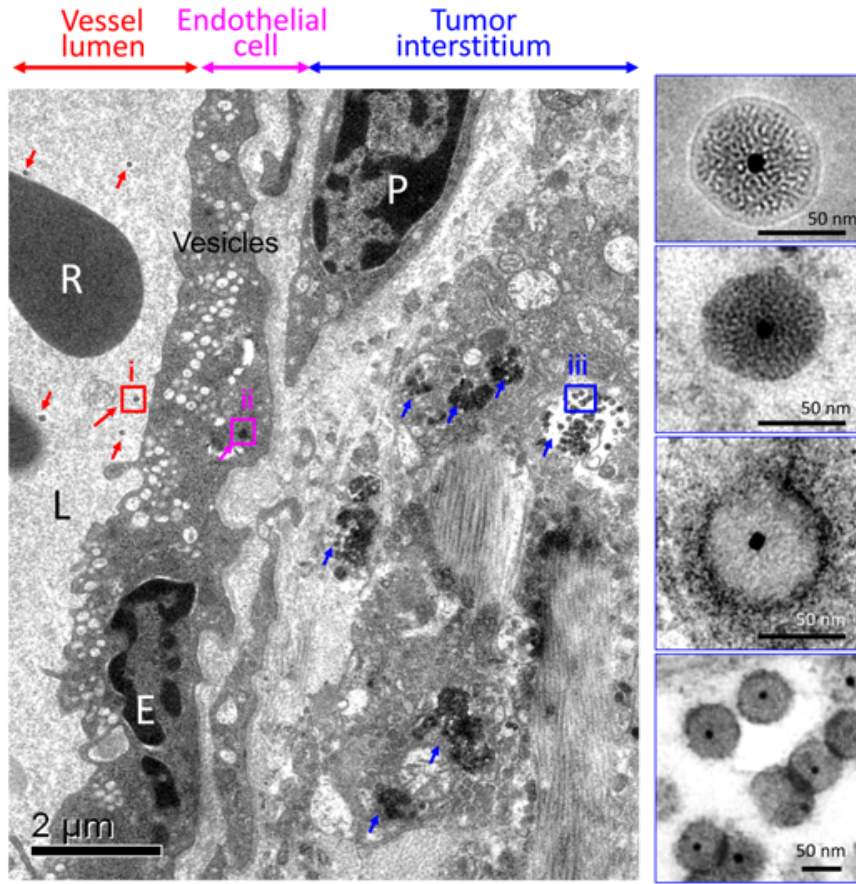

Silicasome with gold core

i: Circulating silicasome

ii: Transcytosing silicasome

iii: Interstitial silicasome

Figure 5. Ultrastructural viewing of the silicasome transport system initiated by iRGD coadministration. (A) Mice bearing orthotopic tumors were injected with $50 \mathrm{mg} / \mathrm{kg} \mathrm{Au}$-silicasomes, with or without coadministration of $8 \mu \mathrm{mol} / \mathrm{kg}$ iRGD. Tumors were harvested at 24 hours and immediately fixed for TEM analysis. At least $10 \mathrm{ROI}$ in each group were viewed to quantitatively express the abundance of grouped, interconnected vesicles (yellow arrows) in the blood vessel endothelial cells. We calculated the number of vesicles per 1 $\mu \mathrm{m}^{2}$ of the intracellular surface area (left panel). Data represent mean \pm SD. ${ }^{*} P<0.05$, 2-tailed Student's $t$ test. Representative TEM pictures with high and low magnification are shown. L, lumen; $\mathrm{R}$, red blood cell. Scale bars: $2 \mu \mathrm{m}$ (left panels); $0.5 \mu \mathrm{m}$ (right panels showing high-magnification images). (B) TEM visualization of silicasome transcytosis in tumor-bearing mice that received $50 \mathrm{mg} / \mathrm{kg} \mathrm{Au}$-silicasome and were sacrificed 24 hours later. The electron micrograph shows silicasomes in (i) the lumen of a tumor blood vessel (red arrows), (ii) transport in the endothelial vesicles (pink arrow), and (iii) deposition in the tumor interstitium (blue arrows). High-magnification images of regions 1 through 3 are provided in the panels on the right. $E$, endothelial cell; $P$, pericyte. Scale bar: $2 \mu \mathrm{m}$ (left panel); $50 \mathrm{~nm}$ (right panels). (C) TEM image showing the presence of silicasomes in a perinuclear distribution inside a cancer cell. N, nucleus; $\mathrm{M}$, mitochondrion. Scale bars: $2 \mu \mathrm{m}$ (left panel); $100 \mathrm{~nm}$ (right panels).
C

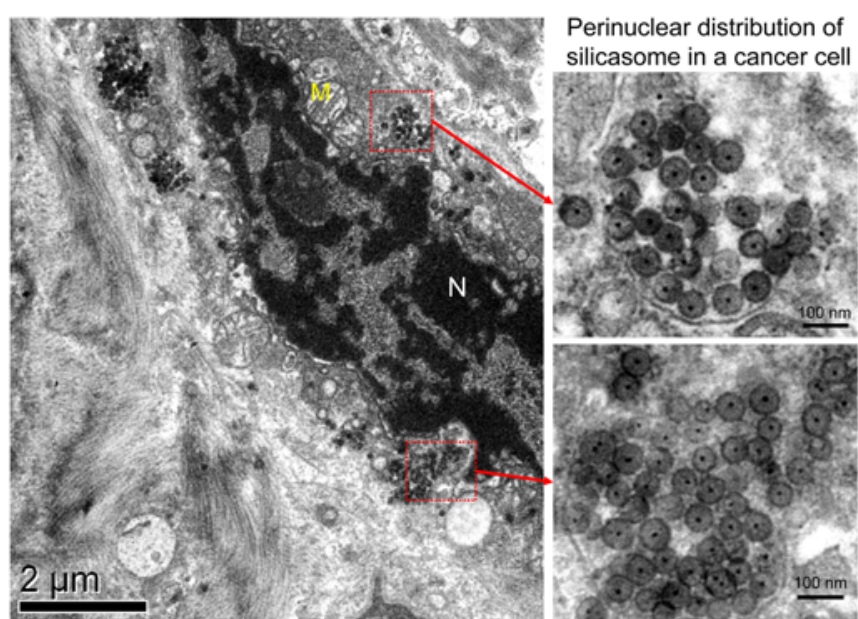


A

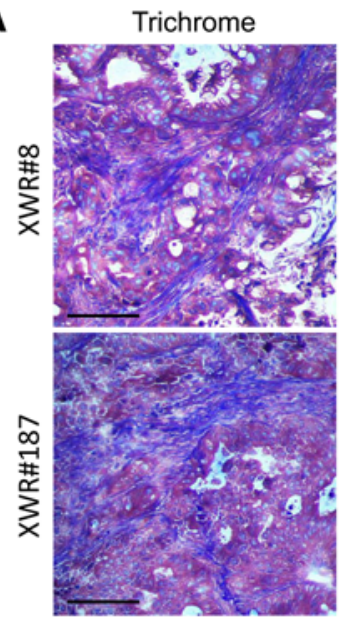

$\mathbf{B}$
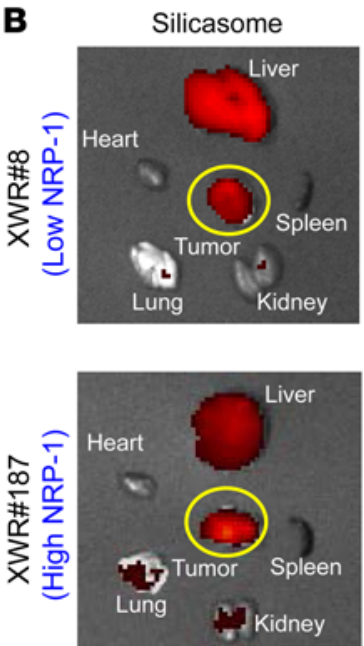

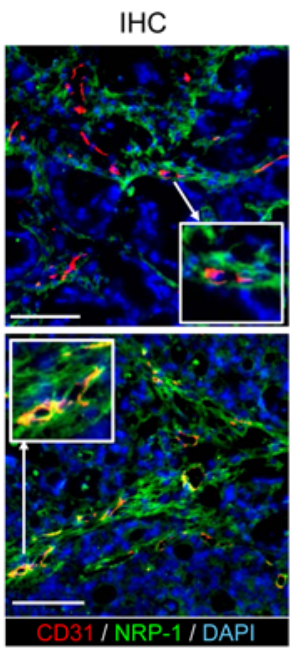

Silicasome + iRGD
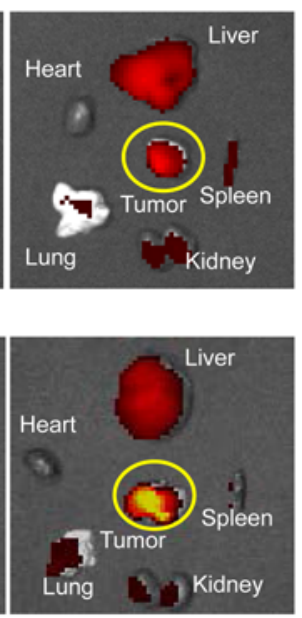
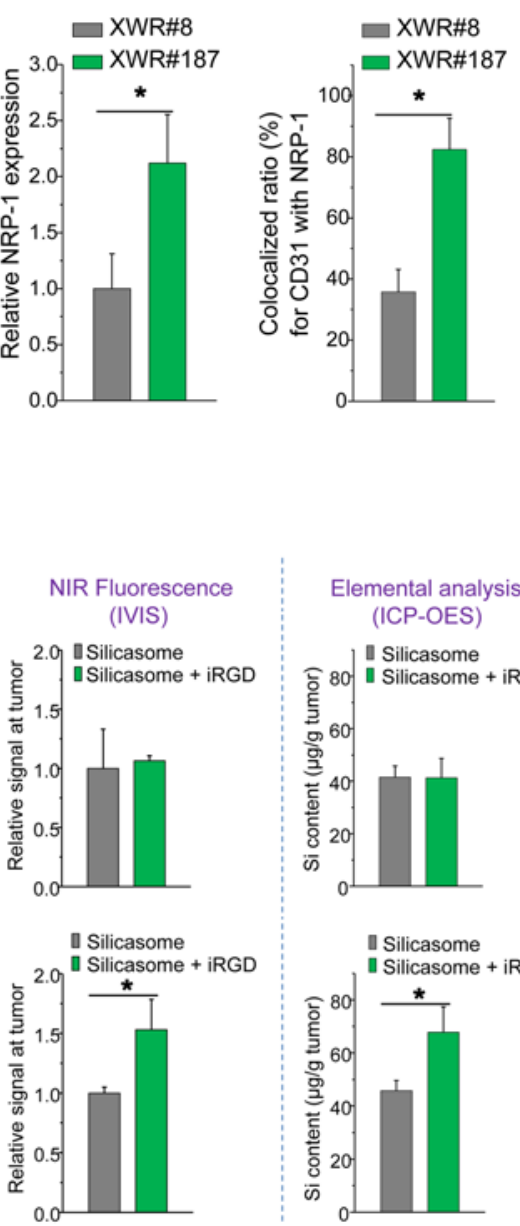

Figure 6. iRGD-induced silicasome biodistribution in patient-derived xenografts in NSG mice. (A) A pair of tumors (XWR\#8 and XWR\#187) with matched stromal abundance but differing levels of NRP-1 expression was selected for a biodistribution study in the absence and presence of iRGD coadministration. Masson's trichrome staining shows equivalent levels of collagen expression in both tumors. Multicolor IHC staining (green fluorescent antibody for NRP-1, red fluorescent antibody for CD31) was used to determine the relative abundance of NRP-1 expression and the extent of overlap with endothelial cells, using ImageJ software. Data represent mean $\pm \mathrm{SD}$. ${ }^{*} P<0.05$, 2-tailed Student's $t$ test. (B) The tumor-bearing animals received i.v. injection of $50 \mathrm{mg} /$ $\mathrm{kg} \mathrm{NIR-labeled} \mathrm{silicasome} \mathrm{with} \mathrm{or} \mathrm{without}$ coadministration of $8 \mu \mathrm{mol} / \mathrm{kg}$ iRGD. Animals were sacrificed after 24 hours $(n=3)$. Ex vivo assessment of the uptake of silicasomes as determined by NIR fluorescence intensity and $\mathrm{Si}$ content. Data represent mean $\pm \mathrm{SD}$. ${ }^{*} P<0.05$, 2-tailed Student's $t$ test. the vesiculo-vacuolar organelle (VVO) described by Dvorak et al. (35). Semiquantitative analysis of vesicle density, determined by counting the number of vesicles in at least 10 regions of interest (ROIs) and expressing the vesicle number per $\mu \mathrm{m}^{2}$ of interior surface area in the cell, demonstrated that iRGD can increase vesicle density approximately 3-fold compared with that of animals not receiving peptide coadministration (Figure $5 \mathrm{~A}$ ).

When attempting to visualize silicasome transport by the vesicular system, the low electron density of the MSNPs is difficult to visualize in the heterogeneous and complex PDAC microenvironment. To address this challenge, silicasomes were synthesized to include an approximately $10 \mathrm{~nm}$ Au-nanoparticle that could be readily visualized by TEM (Figure 1A) (36). Mice expressing orthotopic KPC tumors were i.v. injected with $50 \mathrm{mg} / \mathrm{kg} \mathrm{Au}$-silicasomes in the absence or presence of $8 \mu \mathrm{mol} / \mathrm{kg}$ iRGD. Tumor tissue was harvested 24 hours after injection and fixed to perform TEM analysis. A representative electron micrograph displaying, in one image, (a) electron-dense silicasomes in the blood vessel lumen, (b) vesicular transport in endothelial cells, and (c) particle deposition in the tumor matrix of an animal receiving iRGD coadministration is shown in Figure 5B. Higher magnifications of regions 1-3 in the image confirm the presence of Au-containing particles. It was also possible during iRGD coadministration to demonstrate the appearance of silicasomes in a perinuclear distribution in tumor cells undergoing apoptosis (Figure 5C). While we do not know the exact reason for the intracellular distribution pattern, this seems to be a common characteristic for a variety of chemotherapeutic nanocarriers including MSNPs. This site was several hundred micrometers away from the nearest tumor blood vessel (Figure 5C). While it was possible to observe lower particle density in the tumor matrix of animals not receiving iRGD treatment, we could not locate silicasomes being carried by transcytosed vesicles (Supplemental Figure 6).

Differential impact of $i R G D$ coadministration on silicasome uptake in patient-derived PDAC tumors, phenotyped for NRP-1 expression. While it is clear that the NRP-1 pathway can be functionally engaged by iRGD in the KPC tumor model, we were interested to see whether the peptide could affect silicasome uptake in patient-derived xenografts growing in NOD SCID IL- $2 \alpha$ knockout (NSG) mice (37). One of us (T. Donahue) has established a repository of 23 human PDAC tumors in NSG mice; these tumor samples were obtained from patients during Whipple's surgery. Transferred tumor tissues were phenotypically characterized for cancer features that are distinctive of the corresponding human PDAC tumors, including stromal abundance and the expression of 
oncogenes and signal pathway components that are characteristic of PDAC (37). We used the phenotyping information to select a tumor pair that showed roughly the same stromal abundance, but differed with respect to the density and distribution of NRP-1 expression, as determined by IHC staining. The staining was performed on tumor tissue harvested from NSG mice at the third or fourth tumor passage, using ImageJ software to compare the density of NRP-1 expression as well as its colocalization with CD31 and the cellular nucleus (DAPI) (Figure 6). This led to the identification of the tumor samples, designated XWR\#8 and XWR\#187, that displayed similar collagen density (trichrome staining), but differed in NRP-1 expression (Figure 6A). Thus, while XWR\#8 was characterized by low NRP-1 abundance, XWR\#187 had high NRP-1 expression levels (Figure 6A). XWR\#187 also exhibited more NRP-1-positive tumor blood vessels ( 80\%) compared with XWR\#8 ( $35 \%)$. Subcutaneous xenografts $(n=3)$ were established on the flank of NSG mice before the animals were i.v. injected with NIR-labeled silicasomes $(50 \mathrm{mg} / \mathrm{kg})$, with or without coadministration of the iRGD peptide $(8 \mu \mathrm{mol} / \mathrm{kg})$. Following animal sacrifice after 24 hours, IVIS imaging of the explanted tissues showed a $50 \%$ increase of NIR intensity at the tumor site of XWR\#187 mice receiving iRGD; similar enhancement was not seen in XWR\#8 mice (Figure 6B). These data were confirmed by assessing the elemental Si content of the tumor tissues using ICP-OES (Figure 6B). All things considered, our data indicate that the density and distribution of NRP-1 expression determine the degree of silicasome biodistribution to human tumors in vivo.

\section{Discussion}

In this communication, we demonstrated that the efficacy of an irinotecan-loaded silicasome carrier can be markedly improved by the coadministration of an unconjugated iRGD peptide that does not require attachment to the carrier. Coadministration of the free iRGD peptide increased silicasome uptake at orthotopic KPC tumors sites 3- to 4-fold, leading to enhanced killing of the primary tumor as well as metastasis inhibition. Overall, this resulted in a substantial improvement in animal survival over the irinotecan-loaded silicasome alone $(P=0.027)$. The iRGD effect is mediated by interaction with tumor-associated integrins initially, followed by peptide cleavage and the release of the C-terminal end that engages NRP-1 $(17,19)$. Although the physiological role of NRP-1 is to control transcytosis for nutritional purposes, the vesicular system can also be used for the transport of nanoparticles, as demonstrated by the reduction of particle transport after the injection of receptor-blocking antibodies. Moreover, EM imaging provided ultrastructural evidence that iRGD could induce the appearance of grouped vesicles in endothelial cells, with the ability to carry Au-labeled silicasomes from the blood vessel lumen to the tumor matrix. We also obtained evidence that NRP-1 regulates a transcytosis pathway in human pancreatic tumors that have been implanted in NSG mice. The selection of a tumor pair with differential NRP-1 expression on the tumor vasculature demonstrated differences in carrier uptake and irinotecan delivery during iRGD treatment. All things considered, these data indicate that it is possible to contemplate the use of a personalized approach to PDAC chemotherapy to enhance the efficacy of irinotecan-loaded silicasome carriers by iRGD coadministration.
The utility of a transcytosis pathway to enhance irinotecan delivery in PDAC is impactful for a number of reasons. The first is the display of a dysplastic stroma, which contributes to drug resistance at the tumor site in addition to augmenting tumor growth and metastasis $(12,13)$. While it is often understood that abnormal vascular permeability is the reason for the nanoparticle extravasation, a concept referred to as the EPR effect, we know that the pancreatic cancer stroma actively interferes in vascular permeability $(16,38-40)$. This includes the presence of pericytes that tightly adhere to vascular endothelial cells $(16,38-$ 40). Thus, while the EPR effect may contribute to the nanocarrier uptake in PDAC, it is important to consider the possibility that other vascular mechanisms may add to the nanoparticle uptake at the tumor site, including the possible contribution of nutritional transport pathways and vascular growth factors, which regulate the nutritional as well as vascular permeability mechanisms $(4,14,31,35,41,42)$. The Ruoslahti laboratory described an endocytic pathway that suggests a role in tumor nutrient transport and can also be therapeutically engaged by tumor-penetrating iRGD peptides (21). In addition, vascular growth factors, such as VEGF, VEGF-A, VEGF-A165, TGF- $\beta$, and semaphorin 3A, display CendR motifs that allow binding to $\alpha_{v} \beta_{3}$ and $\alpha_{v} \beta_{5}$ integrins on the tumor vasculature $(18,19,21)$. Thus, proteolytic cleavage and release of the CendR motif could trigger NRP-1-mediated transcytosis (21) in addition to the role of vascular growth factor signaling pathways and their ability to control vascular permeability (43-45). Consequently, it is possible that the NRP-1 pathway may coexist with vascular leakage, including the EPR effect, but displaying different time kinetics. While the response to the CendR motif may commence within minutes, the EPR effect typically requires 6 to 8 hours to peak $(17,46)$.

Our data for silicasome transcytosis during iRGD coadministration supplement previous attempts to overcome the stromal-vascular barrier during PDAC treatment $(12,13,16,26)$. Several agents have been introduced to improve tumor permeability that could be applied to enhancing the tumor access of nanocarriers $(12,42)$. Among these, a hedgehog pathway inhibitor (IPI926) was shown to provide a temporary increase in PDAC vascular density (32) and a PEGylated human recombinant PH2O hyaluronidase (PEGPH20) was shown to induce the reexpansion of PDAC blood vessels (47). Other agents that promote vascular permeability at the tumor site include bradykinin, nitric oxide, angiotensin-converting enzyme inhibitors, TNF- $\alpha$, heme oxygenase-1, collagenase, and hyaluronidase (42, 48-53). We have also demonstrated that the use of a nanocarrier that delivers a small molecule inhibitor of the TGF- $\beta$ pathway, LY364947, can rapidly ( $<2$ hours) reverse pericyte adherence to endothelial cells in vivo (16). The accompanying multiple fold increase in vascular permeability was demonstrated to provide a dramatic increase in the egress of gemcitabine-delivering liposomes at the tumor site (19). Other approaches for enhancing blood vessel permeability by targeting of the TGF- $\beta$ pathway have also been reported $(39,40)$. Finally, it is worth mentioning that the silicasome carrier can achieve stromal reduction by codelivery of paclitaxel with gemcitabine (26), to the extent that our carrier could outperform the administration of Abraxane plus free gemcitabine during treatment of orthotopic PDAC tumors in mice (54). 
Based on the demonstration that codelivery of iRGD enhances the uptake of silicasomes at the PDAC tumor site (Figure 2), it is important to stress that the coadministration approach overcomes a major limitation of the alternative delivery mechanism where the peptide is conjugated to the nanocarrier. The explanation lies in the transport capacity of the carrier system, based on the available number of NRP-1 receptors $(19,20)$. Thus, while the transport of the conjugated silicasome is limited by the relatively small and finite number of target receptors on the vasculature, separate injection of the unconjugated peptide triggers bulk transfer of bystander silicasomes (in greater number) at the tumor site. Moreover, free iRGD also harbors antimetastatic activity through the regulation of integrin function, as demonstrated by interference in the attachment and migration of culture tumor cells on a fibronectin matrix (55). This could explain, in part, the peptide interference on tumor metastasis in our study (Figure 3, B and C). The use of the free peptide is also more practical and affordable for clinical use, as compared with relying on a conjugation mechanism that increases the cost and the complexity of the carrier synthesis.

\section{Methods}

A more detailed description of materials and experimental procedures appears in the Supplemental Materials and Methods.

\section{Silicasome preparation}

Synthesis of irinotecan-loaded silicasome. The 65-nm MSNP core was synthesized using a sol-gel procedure as described before (27). A lipid biofilm was used to produce the silicasomes as previously reported $(26,27)$. Briefly, $500 \mathrm{mg}$ MSNPs were soaked in a $20 \mathrm{ml}$ $\mathrm{TEA}_{8} \mathrm{SOS}(80 \mathrm{mM})$ solution, which was added on top of the lipid biofilm, composed of a $550 \mathrm{mg}$ mixture of DSPC/Chol/DSPE-PEG ${ }_{2000}$ (molar ratio 3:2:0.15). This mixture was coated at the bottom of a round-bottom flask (27). After sonication to accomplish particle coating with an LB, free $\mathrm{TEA}_{8}$ SOS was removed by size-exclusion chromatography over a Sepharose CL-4B column. The TEA SOSloaded silicasomes were incubated in a $10 \mathrm{mg} / \mathrm{ml}$ irinotecan solution for drug loading in a water bath at $65^{\circ} \mathrm{C}$. The loading of the silicasomes with the irinotecan solution was stopped after 30 minutes by quenching the silicasomes in an ice-water bath, after which the drug-loaded silicasomes were washed 3 times by centrifugation and resuspended in PBS.

Synthesis of iRGD-conjugated silicasome. iRGD-conjugated silicasomes were synthesized by linking the peptide to a PEG chain included in the LB. This was accomplished by using commercially available DSPE-PEG ${ }_{2000}$-maleimide in place of DSPE-PEG ${ }_{2000}$, while maintaining the same molar ratio of the lipids as described above. An excess $(0.15 \mathrm{ml}, 5 \mathrm{mg} / \mathrm{ml})$ of the cysteine-modified iRGD peptide was conjugated to the DSPE-PEG ${ }_{2000}$-maleimide, using a thiol-maleimide reaction carried out at room temperature for 4 hours (17). The particles were washed to remove the nonreacted iRGD. The success of the conjugation reaction was confirmed by also preparing a batch of particles conjugated to a fluorescein-labeled (FAM-labeled) iRGD peptide, followed by extensive washing (17) (Supplemental Figure 1A).

Synthesis of silicasomes with an Au core marker. Au nanoparticles of approximately $10 \mathrm{~nm}$ were made by adding $5 \mathrm{ml} \mathrm{HAuCl}_{4}(10 \mathrm{mM})$ and $45 \mathrm{ml}$ Milli-Q water to a $100 \mathrm{ml}$ round-bottom flask equipped with a condenser. After the solution reached boiling temperature while being stirred vigorously, $5.8 \mathrm{ml}$ of sodium citrate $(38.8 \mathrm{mM})$ was added, resulting in a color change from pale yellow to burgundy. The boiling solution was stirred for 10 minutes at $160^{\circ} \mathrm{C}$ and then stirred for an additional 15 minutes without heating. To grow the MSNP shell on the Au nanoparticle core, $36 \mathrm{ml}$ of the citrate-capped particles was rapidly injected into $12 \mathrm{ml} \mathrm{CTAC}$ solution (25 wt\% in $\mathrm{H}_{2} \mathrm{O}$ ). The particles were washed and resuspended in a CTAC solution $\left(6.25 \mathrm{wt} \%\right.$ in $\left.\mathrm{H}_{2} \mathrm{O}\right)$, with stirring at $350 \mathrm{rpm}$ for 5 minutes at $85^{\circ} \mathrm{C}$. To this mixture, we added $0.256 \mathrm{ml}$ of $10 \%(\mathrm{w} / \mathrm{v})$ triethanolamine for 10 minutes, followed by dropwise addition of $0.32 \mathrm{ml}$ of the silica precursor TEOS. The solution was stirred at $350 \mathrm{rpm}$ for 20 minutes, leading to the creation of Au core/MSNP shell particles with an average size of approximately $65 \mathrm{~nm}$. The particles were purified by sequential washing in $1 \% \mathrm{NaCl}$ in methanol (w/v) and pure methanol. The Au-labeled MSNPs were then coated with an LB, as described above.

\section{Biodistribution study of i.v.-injected silicasomes with or without iRGD coadministration}

IVIS (Xenogen) imaging was used to study the biodistribution of NIR-labeled silicasomes in the KPC-derived orthotopic model $(n=$ 3 mice/group) (27). Animals were i.v. injected with $50 \mathrm{mg} / \mathrm{kg}$ of the conjugated and nonconjugated silicasomes with or without the coadministration of $8 \mu \mathrm{mol} / \mathrm{kg}$ iRGD. For coadministration, free iRGD peptide was mixed with the silicasomes in one syringe immediately before injection. Animals were sacrificed after 24 hours followed by ex vivo imaging of the excised tumors and major organs. Tumor biodistribution was also confirmed by assessing the Si content using an ICP-OES protocol (27).

\section{Assessment of irinotecan-loaded silicasome efficacy by iRCD coadministration in the KPC-derived orthotopic tumor model}

Tumor-bearing B6/129 mice were randomly assigned into 4 groups, with 6 animals each. The first group was i.v. injected with silicasomes containing an irinotecan dose of $40 \mathrm{mg} / \mathrm{kg}(80 \mathrm{mg} / \mathrm{kg}$ MSNP) every 3 days, for a total of 4 administrations. The second group received the same dose of the irinotecan-loaded silicasome plus coadministration of $8 \mu \mathrm{mol} / \mathrm{kg}$ iRGD. The third and fourth groups were treated with PBS or iRGD alone. The mice were monitored daily up to the point of spontaneous animal death or approaching moribund status $(27,32)$. Bioluminescence imaging of the primary tumor and metastasis sites was performed by injecting the animals i.p. with $75 \mathrm{mg} /$ $\mathrm{kg}$ D-luciferin, 10 minutes before sacrifice. The tumor tissue and major organs (gastrointestinal tract, liver, spleen, heart, lung, and kidneys) were harvested for quantitative assessment of bioluminescence image intensity.

\section{Ultrastructural analysis of the transcytosis pathway through TEM viewing}

KPC-derived orthotopic tumor-bearing mice were treated by i.v. injection of $50 \mathrm{mg} / \mathrm{kg}$ of the Au-encapsulated silicasomes, with or without the coadministration of $8 \mu \mathrm{mol} / \mathrm{kg}$ iRGD. Tumor biopsies were collected after 24 hours, washed in PBS, and immediately fixed at $4^{\circ} \mathrm{C}$ with $2.5 \%$ glutaraldehyde. Further sample preparation and sectioning were performed by the Electron Microscopy Services Center at UCLA. After fixation in $1 \% \mathrm{OsO}_{4}$, the samples were dehy- 
drated in propylene oxide and embedded in resin. Tissue slices 60 to $80 \mathrm{~nm}$ thick were placed on copper grids and viewed under a JEOL 1200-EX electron microscope.

\section{Silicasome biodistribution in patient-derived PDAC tumors}

A repository of 23 PDAC samples was collected from patients undergoing Whipple's surgery and used to establish xenografts in NSG mice in T. Donahue's laboratory. Utilizing phenotyping data and performance of IHC staining for NRP-1 expression, 2 patient samples (XWR\#8 and XWR\#187) were collected for growing fresh subcutaneous xenografts in the flanks of 6-week-old female NSG mice (37). When the tumor size grew to a diameter of approximately $0.8 \mathrm{~cm}, 3$ animals in each group receiving silicasomes, with or without iRGD coadministration, were used to assess biodistribution to the tumor site, similarly to the procedure described above. Animals were sacrificed after 24 hours for the performance of ex vivo imaging to determine the uptake of NIR-labeled silicasomes. The imaging data were also confirmed by assessing the Si content at the tumor sites by ICP-OES.

\section{Statistics}

Comparative analysis of differences between groups was performed using the 2-tailed Student's $t$ test (Excel software, Microsoft) or 1-way ANOVA followed by Tukey's multiple comparisons (Origin software, OriginLab). Values were expressed as mean \pm SD of multiple determinations, as stated in the figure legends. The survival data were processed by the log-rank test (Mantel-Cox) using SPSS software. For all statistical analyses, $P<0.05$ was considered statistically significant.

\section{Study approval}

All animal experiments were performed using protocols approved by the UCLA Animal Research Committee. UCLA Institutional Review Board approval was given for collection of samples from human subjects and informed consent was given by all participating human subjects.

\section{Author contributions}

XL, AEN, and HM designed research. XL, PL, IP, JL, YPL, NW, $\mathrm{JJ}$, and CHC performed research. TD provided the patient tumor model. ZW provided clinical consultation. XL and HM analyzed data. XL, AEN, and HM wrote the paper.

\section{Acknowledgments}

This study was funded by U.S. Public Health Service Grant 1U01CA198846. The project also received partial support from the Hirshberg Foundation for Pancreatic Cancer Research. We thank Venkata Ramana Kotamraju and Erkki Ruoslahti for providing reactive iRGD peptide and anti-NRP-1 antibody.

Address correspondence to: Huan Meng, Department of Medicine, Division of NanoMedicine, University of California, Los Angeles, 570 Westwood Plaza, Building 114, Room 6511, Los Angeles, California 90095, USA. Phone: 310.825.0217; E-mail: hmeng@mednet.ucla.edu. Or to: Andre E. Nel, Department of Medicine, UCLA Med-Nano Medicine 10833 Le Conte Avenue, 52-175 CHS Los Angeles, California 90095, USA. Phone: 310.825.6620; E-mail: anel@mednet.ucla.edu.
1. Peer D, Karp JM, Hong S, Farokhzad OC, Margalit R, Langer R. Nanocarriers as an emerging platform for cancer therapy. Nat Nanotechnol. 2007;2(12):751-760.

2. Davis ME, Chen ZG, Shin DM. Nanoparticle therapeutics: an emerging treatment modality for cancer. Nat Rev Drug Discov. 2008;7(9):771-782.

3. Giljohann DA, Seferos DS, Daniel WL, Massich MD, Patel PC, Mirkin CA. Gold nanoparticles for biology and medicine. Angew Chem Int Ed Engl. 2010;49(19):3280-3294.

4. Ruoslahti E, Bhatia SN, Sailor MJ. Targeting of drugs and nanoparticles to tumors. JCell Biol. 2010;188(6):759-768.

5. Roco MC, Mirkin CA, Hersam MC. Nanotechnology research directions for societal needs in 2020: summary of international study.

J Nanopart Res. 2011;13(3):897-919.

6. Ma M, Chen H, Shi J. Construction of smart inorganic nanoparticle-based ultrasound contrast agents and their biomedical applications. Sci Bull. 2015;60(13):1170-1183.

7. Von Hoff DD, et al. Increased survival in pancreatic cancer with nab-paclitaxel plus gemcitabine. N Engl JMed. 2013;369(18):1691-1703.

8. Wang-Gillam A, et al. Nanoliposomal irinotecan with fluorouracil and folinic acid in metastatic pancreatic cancer after previous gemcitabine-based therapy (NAPOLI-1): a global, randomised, open-label, phase 3 trial. Lancet. 2016;387(10018):545-557.

9. Li F, et al. Multiple layer-by-layer lipid-polymer hybrid nanoparticles for improved FOLFIRINOX chemotherapy in pancreatic tumor models. Adv Funct Mater. 2015;25(5):788-798.

10. Pan Y, et al. Gd-metallofullerenol nanomaterial suppresses pancreatic cancer metastasis by inhibiting the interaction of histone deacetylase 1 and metastasis-associated protein 1. ACS Nano. 2015;9(7):6826-6836.

11. Han $\mathrm{H}$, et al. Theranostic reduction-sensitive gemcitabine prodrug micelles for near-infrared imaging and pancreatic cancer therapy. Nanoscale. 2016;8(1):283-291.

12. Dimou A, Syrigos KN, Saif MW. Overcoming the stromal barrier: technologies to optimize drug delivery in pancreatic cancer. Ther Adv Med Oncol. 2012;4(5):271-279.

13. Feig C, Gopinathan A, Neesse A, Chan DS, Cook N, Tuveson DA. The pancreas cancer microenvironment. Clin Cancer Res. 2012;18(16):4266-4276.

14. Maeda H, Wu J, Sawa T, Matsumura Y, Hori K. Tumor vascular permeability and the EPR effect in macromolecular therapeutics: a review. JControl Release. 2000;65(1-2):271-284.

15. Chauhan VP, Stylianopoulos T, Boucher Y, Jain RK. Delivery of molecular and nanoscale medicine to tumors: transport barriers and strategies. Annu Rev Chem Biomol Eng. 2011;2:281-298.

16. Meng H, et al. Two-wave nanotherapy to target the stroma and optimize gemcitabine delivery to a human pancreatic cancer model in mice. ACS Nano. 2013;7(11):10048-10065.

17. Sugahara KN, et al. Tissue-penetrating delivery of compounds and nanoparticles into tumors. Cancer Cell. 2009;16(6):510-520.
18. Teesalu T, Sugahara KN, Kotamraju VR, Ruoslahti E. C-end rule peptides mediate neuropilin-1-dependent cell, vascular, and tissue penetration. Proc Natl Acad Sci US A. 2009;106(38):16157-16162.

19. Sugahara KN, et al. Coadministration of a tumor-penetrating peptide enhances the efficacy of cancer drugs. Science. 2010;328(5981):1031-1035.

20. Ruoslahti E. Peptides as targeting elements and tissue penetration devices for nanoparticles. $A d v$ Mater Weinheim. 2012;24(28):3747-3756.

21. Pang HB, et al. An endocytosis pathway initiated through neuropilin-1 and regulated by nutrient availability. Nat Commun. 2014;5:4904.

22. Akashi Y, et al. Anticancer effects of gemcitabine are enhanced by co-administered iRGD peptide in murine pancreatic cancer models that overexpressed neuropilin-1. Br J Cancer. 2014;110(6):1481-1487.

23. Suh WH, Suh Y-H, Stucky GD. Multifunctional nanosystems at the interface of physical and life sciences. Nano Today. 2009;4(1):27-36.

24. Meng $\mathrm{H}$, et al. Autonomous in vitro anticancer drug release from mesoporous silica nanoparticles by pH-sensitive nanovalves. J Am Chem Soc. 2010;132(36):12690-12697.

25. Ambrogio MW, Thomas CR, Zhao YL, Zink JI, Stoddart JF. Mechanized silica nanoparticles: a new frontier in theranostic nanomedicine. Acc Chem Res. 2011;44(10):903-913.

26. Meng H, et al. Use of a lipid-coated mesoporous silica nanoparticle platform for synergistic gemcitabine and paclitaxel delivery to human pancreatic 
cancer in mice. ACS Nano. 2015;9(4):3540-3557.

27. Liu X, et al. Irinotecan delivery by lipid-coated mesoporous silica nanoparticles shows improved efficacy and safety over liposomes for pancreatic cancer. ACS Nano. 2016;10(2):2702-2715.

28. Tseng WW, et al. Development of an orthotopic model of invasive pancreatic cancer in an immunocompetent murine host. Clin Cancer Res. 2010;16(14):3684-3695.

29. Torres MP, Rachagani S, Souchek JJ, Mallya K, Johansson SL, Batra SK. Novel pancreatic cancer cell lines derived from genetically engineered mouse models of spontaneous pancreatic adenocarcinoma: applications in diagnosis and therapy. PLoS One. 2013;8(11):e80580.

30. Hussain S, Rodriguez-Fernandez M, Braun GB, Doyle FJ, Ruoslahti E. Quantity and accessibility for specific targeting of receptors in tumours. Sci Rep. 2014;4:5232.

31. Li SD, Huang L. Pharmacokinetics and biodistribution of nanoparticles. Mol Pharm. 2008;5(4):496-504.

32. Olive KP, et al. Inhibition of Hedgehog signaling enhances delivery of chemotherapy in a mouse model of pancreatic cancer. Science. 2009;324(5933):1457-1461.

33. Ruoslahti E, Pierschbacher MD. New perspectives in cell adhesion: RGD and integrins. Science. 1987;238(4826):491-497.

34. Hanahan D, Weinberg RA. The hallmarks of cancer. Cell. 2000;100(1):57-70.

35. Feng D, Nagy JA, Hipp J, Dvorak HF, Dvorak AM. Vesiculo-vacuolar organelles and the regulation of venule permeability to macromolecules by vascular permeability factor, histamine, and serotonin. J Exp Med. 1996;183(5):1981-1986.

36. Liu X, et al. Enhanced retention and cellular uptake of nanoparticles in tumors by controlling their aggregation behavior. ACS Nano.
2013;7(7):6244-6257.

37. Rückert F, et al. Five primary human pancreatic adenocarcinoma cell lines established by the outgrowth method. J Surg Res. 2012;172(1):29-39.

38. Kano MR, et al. Improvement of cancer-targeting therapy, using nanocarriers for intractable solid tumors by inhibition of TGF-beta signaling. Proc Natl Acad Sci U S A. 2007;104(9):3460-3465.

39. Cabral H, et al. Accumulation of sub-100 nm polymeric micelles in poorly permeable tumours depends on size. Nat Nanotechnol. 2011;6(12):815-823.

40. Liu J, et al. TGF- $\beta$ blockade improves the distribution and efficacy of therapeutics in breast carcinoma by normalizing the tumor stroma. Proc Natl Acad Sci U S A. 2012;109(41):16618-16623.

41. Jain RK, Stylianopoulos T. Delivering nanomedicine to solid tumors. Nat Rev Clin Oncol. 2010;7(11):653-664.

42. Kobayashi H, Watanabe R, Choyke PL. Improving conventional enhanced permeability and retention (EPR) effects; what is the appropriate target? Theranostics. 2013;4(1):81-89.

43. Kolodkin AL, Levengood DV, Rowe EG, Tai YT, Giger RJ, Ginty DD. Neuropilin is a semaphorin III receptor. Cell. 1997;90(4):753-762.

44. Ellis LM. The role of neuropilins in cancer. $\mathrm{Mol}$ Cancer Ther. 2006;5(5):1099-1107.

45. Glinka Y, Prud'homme GJ. Neuropilin-1 is a receptor for transforming growth factor beta-1, activates its latent form, and promotes regulatory T cell activity. J Leukoc Biol. 2008;84(1):302-310.

46. Maeda H, Fang J, Inutsuka T, Kitamoto Y. Vascular permeability enhancement in solid tumor: various factors, mechanisms involved and its implications. Int Immunopharmacol. 2003;3(3):319-328.

47. Jacobetz MA, et al. Hyaluronan impairs vascular function and drug delivery in a mouse model of pancreatic cancer. Gut. 2013;62(1):112-120.

48. Eikenes L, Bruland ØS, Brekken C, Davies Cde

L. Collagenase increases the transcapillary pressure gradient and improves the uptake and distribution of monoclonal antibodies in human osteosarcoma xenografts. Cancer Res. 2004;64(14):4768-4773.

49. Seynhaeve AL, et al. Tumor necrosis factor alpha mediates homogeneous distribution of liposomes in murine melanoma that contributes to a better tumor response. Cancer Res. 2007;67(19):9455-9462.

50. Fang J, Nakamura H, Maeda H. The EPR effect: Unique features of tumor blood vessels for drug delivery, factors involved, and limitations and augmentation of the effect. Adv Drug Deliv Rev. 2011;63(3):136-151.

51. Fang J, Qin H, Nakamura H, Tsukigawa K, Shin T, Maeda H. Carbon monoxide, generated by heme oxygenase-1, mediates the enhanced permeability and retention effect in solid tumors. Cancer Sci. 2012;103(3):535-541.

52. Maeda H. The link between infection and cancer: tumor vasculature, free radicals, and drug delivery to tumors via the EPR effect. Cancer Sci. 2013;104(7):779-789.

53. Eikenes L, Tari M, Tufto I, Bruland OS, de Lange Davies C. Hyaluronidase induces a transcapillary pressure gradient and improves the distribution and uptake of liposomal doxorubicin (Caelyx) in human osteosarcoma xenografts. Br J Cancer. 2005;93(1):81-88.

54. Frese KK, et al. nab-Paclitaxel potentiates gemcitabine activity by reducing cytidine deaminase levels in a mouse model of pancreatic cancer. Cancer Discov. 2012;2(3):260-269.

55. Sugahara KN, et al. Tumor-penetrating iRGD peptide inhibits metastasis. Mol Cancer Ther. 2015;14(1):120-128. 